\title{
Targeting historically refractory interfaces: a partnership model that accelerates drug discovery within an expanded haystack
}

"While drug-discovery professionals view the concept of an expanded druggable genome as exciting, it is unlikely that these findings will significantly impact US FDA approval rates for new molecular entities in the near future..."

The completion of the Human Genome Project has resulted in the identification of approximately 30,000 genes, and a subset of these genes, estimated to be approximately 3000-10,000, are believed to be involved in the pathogenesis of human disease [1]. This and other studies have contributed to the wealth of convergent 'omic' data, which have caused many to speculate on the contribution of these genes to the concept of a 'changing discovery landscape' [1-7]. Several comprehensive reviews evaluating novel target space have focused on the concept of an expanding 'druggable genome', which has resulted in recalibrated estimates of the number of druggable targets at approximately 300-6000, based on the definition of 'druggable' used in the respective study [8-14]. While drug-discovery professionals view the concept of an expanded druggable genome as exciting, it is unlikely that these findings will significantly impact US FDA approval rates for new molecular entities (NMEs) in the near future, which is troubling for many. In 2008, there were only 21 approved NMEs and, while this represents a $20 \%$ increase over 2007, these data are sobering given imminent patent concerns, the estimated penetrance of generics into the market place and the increasing competition within the limited target landscape that many pharmaceutical companies are facing [15-17]. One tenet put forward to explain the current paucity of NMEs that has plagued the drug-discovery and development community for almost a decade is a lack of innovative/creative exploration, with many 'looking under the same lamp post for lost keys' $[18,19]$. In support of this 'me too' mantra several large pharmaceutical companies have reinforced their reluctance to pursue targets within this expanded definition of 'druggable space' on the basis of

historical metrics suggesting many of these targets are intractable to orally bioavailable small molecules, and that preclinical validation of these targets will be challenging if not unlikely $[20,21]$. In establishing disease causality and validating this novel space as therapeutically druggable, several groups have partnered structural biology data with extensive systems biology curation studies. Their efforts have elucidated numerous cellular networks and provided systematic protein-protein interactomes that they conclude are requisite in human health and disease [7,22-25]. Integration of these data has recently accelerated a global interest in the therapeutic development of expanded target space from within the newly defined 'druggable haystack' that includes protein-protein interaction interfaces using small molecules [26-28].

\begin{abstract}
"Integration of these data has recently accelerated a global interest in the therapeutic development of expanded target space from within the newly defined 'druggable haystack' that includes protein-protein interaction interfaces using small molecules."
\end{abstract}

Macromolecular complex assemblies comprised of protein-protein and/or protein-DNA interactions are the functional cornerstones of signal transduction, the immune response and other essential cellular processes. These include gene transcription, which is dependent upon specific protein-DNA interactions involving basal and/or gene-specific transcription factors (TFs). While there is mounting evidence articulating varied successes in targeting protein-protein interaction interfaces, we believe that the protein-DNA interaction interface also represents significant opportunities for

\section{Alan C Rigby}

Center for Vascular Biology

Research, Division of

Molecular and Vascular

Medicine, Department of

Medicine, Beth Israel

Deaconess Medical Center,

Harvard Medical School,

Boston, MA 02215, USA

Tel.: + I 6176670637

Fax: + I 6176672913

E-mail: arigby@

bidmc.harvard.edu 
the development of paradigm-altering therapies [27-30]. Along these lines, Aikawa and colleagues recently reported the peptidomimetic discovery of a small-molecule inhibitor of the activator protein (AP)-1 heterodimeric $\mathrm{TF}$, which was identified using a $3 \mathrm{D}$ computer-aided drug discovery (CADD) pharmacophoric model derived from the co-complex structure of the AP-1 TF, bound to a cyclic decapeptide that inhibited DNA binding with an IC $_{50}$ of approximately $64 \mu \mathrm{m}$. Their novel small molecule inhibits inflammatory cytokine release and matrix metalloproteinase (MMP) action in an animal model of rheumatoid arthritis [31]. However, continued success in targeting these historically refractory interfaces will require a synergistic, multidisciplinary, collaborative tour de force of creativity, which leverages the skills, expertise and infrastructure of all partners involved $[19,32,33]$. The concept of selectively targeting and inhibiting the interaction interfaces of protein-protein and/or protein-DNA complexes represents a paradigm shift in therapeutic strategies aimed at reprogramming specific pathways, or programs of gene expression that are deregulated in cancer, inflammation and other complex disease states where there is still considerable unmet therapeutic need. Given the importance and commercial promise of these 'nontraditional' interfacial targets, several groups have attempted to overcome the inherent physiochemical properties of this target space, as well as psychological barriers that have long discouraged the idea of developing therapies for targets within this expanded target space ([26-29,34] and references therein).

\section{"While there is mounting evidence articulating varied successes in targeting protein-protein interaction interfaces, we believe that the protein-DNA interaction interface also represents significant opportunities for the development of paradigm-altering therapies."}

The long-standing tenet that these interfaces are excessively complicated and, thus, 'poorly druggable' was founded on a detailed understanding of protein-protein and protein-DNA interface complexity, as well as extensive knowledge of the relative energetic contributions to these interactions by: interfacial contacts, interface size, geometrical complexity, polarity and conformational plasticity. However, one hypothesis is that, within these complex interfaces, there are 'interaction hot spots' that contribute a disproportionate amount of binding energy to complex formation [35,36]. These hot spots tend to reside on both proteins and are clustered within the interaction interface, thereby contributing in a cooperative manner to the formation of the complex through surface complementarity and significant protein flexibility at the amino acid level [36]. A convergence of important biophysical data supporting this hypothesis has provided researchers with a unique targeting strategy in which small molecules are directed to these localized interaction surfaces and evaluated for activity. This approach has resulted in the identification of small molecule 'hits' for several protein-protein interfaces that are in various stages of preclinical optimization and development [35-39] and are reviewed elsewhere [27-29]. Given that many drug-discovery initiatives have and remain focused on traditional targets, including G-protein-coupled receptors (GPCRs), nuclear receptors, ion channels and enzymes, many of the small-molecule repositories available for screening are biased toward this chemical space and closely adhere to the 'rule of five' principles $[40,41]$. In order to improve hit success rates in experimental or in silico screening campaigns, and to facilitate the identification of small-molecule chemotypes with attributes requisite for hit-through-lead optimization within the expanding 'druggable genome', a comprehensive and innovative medicinal chemistry effort focused on novel synthetic methods to rapidly develop arrays of natural product-like structures, and/or combinatorial bioisostere diversity, approaches that facilitate the exploration of an expanded chemical space are desperately needed [42-44]. This chemistry venture will likely continue to bear considerable fruit, but only if traditional metrics of bioavailability, size and blood-brain penetrance are not the only drivers guiding library design and synthesis [28].

One approach that is likely to be increasingly important in the discovery and validation of novel targets within the expanded 'druggable genome' is CADD [45]. CADD platforms that operate on computer grids/clusters are capable of screening virtual libraries comprised of increasingly large numbers of unique small molecules, and provide opportunities to rapidly screen new and/or expanded small-molecule chemical space [46-51]. CADD approaches 
accelerate hit identification and subsequent lead validation and optimization through in silico computational mining of these $3 \mathrm{D}$ libraries. The in silico libraries amenable to this approach can, and should, be as diverse as possible and representative of chemical space that includes natural product-like compounds. Importantly, structure-based virtual screens (SBVSs), in which a virtual compound library is docked into the 'active/druggable' site, offers several competitive advantages over experimental high-throughput screens (HTS), which are often activity-based assays that are frequently difficult to standardize and suffer from reproducibility and high false-positive rates due to colloidal aggregation as well as other concerns. These target-specific CADD approaches, while not without limitations (i.e., reproducibly scoring and ranking active versus false-positive compounds), allow one to rapidly interrogate any novel interaction space and identify chemotypes of interest while rapidly triaging all other compounds. SBVSs use critical structural descriptors that are extracted from the target protein (alone or in complex with endogenous/native ligand), and defined using biophysical approaches. These pharmacophores guide and filter high-throughput docking (HTD) approaches that evaluate these large chemical repositories for 'hits' that satiate these target-specific descriptors using an approach that is both efficient and cost effective. However, the inherent limitations of scoring algorithms currently used to reproducibly rank identified 'hits' must be overcome [49,52]. Similar to the hurdles we are facing regarding the chemical space represented in the current screening libraries that were developed to modulate the traditional targets noted, many of the scoring algorithms currently implemented in CADD platforms use 'consensus and/or generalized scoring functions' developed with these same targets in mind. To ensure the implementation of CADD in early-stage drug-discovery initiatives aimed at this expanded 'druggable genome' or 'haystack', this must change and efforts are underway to address this important limitation. With that said, the future success of this approach is critically dependent upon the continued convergence of these computational approaches with supportive biophysical, structural and/or biological data, which will interface with a refined medicinal chemistry platform that enables affinity, pharmacokinetic and toxicity metrics to be addressed early in the discovery process. Alone, no single expertise will be sufficient to overcome the scientific and intellectual barriers that have previously precluded discovery and validation of these novel targets.

In summary, moving beyond hit identification and further validating these and/or other targets from within this expanded 'haystack' will require a partnership model of discovery that leverages [46,49,50,53-58]:

- The utility and speed of CADD;

- Molecular pharmacology/chemical genetic mechanism of action/genetic perturbation studies;

- Elegant medicinal chemistry strategies focused around bioisostere diversity and diverse oriented synthesis approaches that enable the synthesis of complex, natural product-like, lead optimized small molecules.

"Given the importance and commercial promise of these 'nontraditional' interfacial targets, several groups have attempted to overcome the inherent physiochemical properties of this target space..."

Ultimately, the success of this discovery process will require validation at the structural level through the implementation of NMR spectroscopy and/or x-ray crystallographic approaches. As I have previously discussed, NMR provides a robust platform to characterize ligand binding and affinity, while simultaneously providing a window through which the structure of the entire target protein or proteins can be observed without the need of an assay [47,59-62]. Importantly, this partnership model and its success and/or failure will rely upon our ability to iteratively integrate these essential skills into this innovative discovery pipeline that is predicated on pushing the boundaries of each experimental approach, and constant communication between all involved. While progress has been made vis-à-vis realizing the therapeutic potential of small-molecule inhibitors that target protein-protein interactions and/or disrupt protein-DNA interactions by targeting the interaction interface of dimeric TFs, much remains to be done. The success of refining these and/or other compounds in hit-through-lead optimization strategies will dictate the fate of this exciting and paradigm-altering journey - a journey that we hope has only just begun. 


\section{Acknowledgements}

Alan C Rigby would like to thank those that have collaborated and/or are currently collaborating within his laboratory. He thanks Marianne Grant for editorial comments on this manuscript.

Financial \& competing interests disclosure The author is grateful to the NIH (AR054442-02), Prostate Cancer Foundation and the Harvard Medical
School Clinical and Translational Award (1 UL1 RR 025758-01) for financial support of his discovery initiatives. The author has no other relevant affliations or financial involvement with any organization or entity with a financial interest in or financial conflict with the subject matter or materials discussed in the manuscript apart from those disclosed.

No writing assistance was utilized in the production of this manuscript.

\section{Bibliography}

1 Venter JC, Adams MD, Myers EW et al. The sequence of the human genome. Science 291, 1304-1351 (2001).

2 Rabelo EM, Hall RS, Loukas A et al. Improved insights into the transcriptomes of the human hookworm Necator americanus - fundamental and biotechnological implications. Biotechnol. Adv. 27, 122-132 (2009).

3 Raman K, Yeturu K, Chandra N. TargetTB: a target identification pipeline for Mycobacterium tuberculosis through an interactome, reactome and genome-scale structural analysis. BMC Syst. Biol. 2, 109 (2008).

4 Billingsley ML. Druggable targets and targeted drugs: enhancing the development of new therapeutics. Pharmacology 82 , 239-244 (2008).

5 Ho RL, Lieu CA. Systems biology: an evolving approach in drug discovery and development. Drugs R D 9, 203-216 (2008).

6 Bansal AT, Barnes MR. Genomics in drug discovery: the best things come to those who wait. Curr. Opin. Drug Discov. Devel. 11, 303-311 (2008).

7 Weigelt J, McBroom-Cerajewski LD, Schapira M, Zhao Y, Arrowmsmith CH. Structural genomics and drug discovery: all in the family. Curr. Opin. Chem. Biol. 12, 32-39 (2008).

8 Wishart DS, Knox C, Guo AC et al. DrugBank: a comprehensive resource for in silico drug discovery and exploration. Nucleic Acids Res. 34, D668-D672 (2006).

9 Overington JP, Al-Lazikani B, Hopkins AL How many drug targets are there? Nat. Rev. Drug Discov. 5, 993-996 (2006).

10 Drews J. Stategic trends in the drug industry. Drug Discov. Today 8, 411-420 (2003).

11 Drews II. Drug discovery today - and tomorrow. Drug Discov. Today 5, 2-4 (2000).

12 Drews J. Drug discovery: a historical perspective. Science 287, 1960-1964 (2000).
13 Drews J. Biotechnology's metamorphosis into a drug discovery industry. Nat. Biotechnol. 16(Suppl.), 22-24 (1998).

14 Hopkins AL, Groom CR. The druggable genome. Nat. Rev. Drug Discov. 1, 727-730 (2002).

15 Sams-Dodd F. Research and market strategy: how choice of drug discovery approach can affect market position. Drug Discov. Today 12, 314-318 (2007).

16 Hughes B. 2007 FDA drug approvals: a year of flux. Nat. Rev. Drug Discov. 7, 107-109 (2008).

17 Hughes B. 2008 FDA drug approvals. Nat. Rev. Drug Discov. 8, 93-96 (2009).

18 Sams-Dodd F. Target-based drug discovery: is something wrong? Drug Discov. Today 10, 139-147 (2005).

19 Sams-Dodd F. Optimizing the discovery organization for innovation. Drug Discov. Today 10, 1049-1056 (2005).

20 Hopkins AL, Groom CR. Target analysis: a priori assessment of druggability. Ernst Schering Res. Found. Workshop 42, 11-17 (2003).

21 Sakharkar MK, Sakharkar KR. Targetability of human disease genes. Curr. Drug Discov. Technol. 4, 48-58 (2007).

22 Yu H, Braun P, Yildirim MA et al. Highquality binary protein interaction map of the yeast interactome network. Science 322, 104-110 (2008).

23 Braun P, Tasan M, Dreze M et al. An experimentally derived confidence score for binary protein-protein interactions. Nat. Methods. 6, 91-97 (2009).

24 Cusick ME, Yu H, Smolyar A et al. Literaturecurated protein interaction datasets. Nat. Methods. 6, 39-46 (2009).

25 Simonis N, Rual JF, Carvunis AR et al. Empirically controlled mapping of the Caenorhabditis elegans protein-protein interactome network. Nat. Methods. 6, 47-54 (2009)

26 Arkin MR, Wells JA. Small-molecule inhibitors of protein-protein interactions: progressing towards the dream. Nat. Rev. Drug Discov. 3 , 301-317 (2004).
27 Berg T. Inhibition of transcription factors with small organic molecules. Curr. Opin. Chem. Biol. 12, 464-471 (2008).

28 Wells JA, McClendon CL. Reaching for high-hanging fruit in drug discovery at protein-protein interfaces. Nature 450, 1001-1009 (2007).

29 Berg T. Small-molecule inhibitors of proteinprotein interactions. Curr. Opin. Drug Discov. Devel. 11, 666-674 (2008).

30 Darnell JE Jr. Transcription factors as targets for cancer therapy. Nat. Rev. Cancer 2, 740-749 (2002).

31 Aikawa Y, Morimoto K, Yamamoto T et al. Treatment of arthritis with a selective inhibitor of c-Fos/activator protein-1. Nat. Biotechnol. 26, 817-823 (2008).

32 Sams-Dodd F. Drug discovery: selecting the optimal approach. Drug Discov. Today 11, 465-472 (2006).

33 Gray NS. Drug discovery through industryacademic partnerships. Nat. Chem. Biol. 2, 649-653 (2006).

34 Brennan P, Donev R, Hewamana S. Targeting transcription factors for therapeutic benefit. Mol. Biosyst. 4, 909-919 (2008).

35 Guney E, Tuncbag N, Keskin O, Gursoy A. HotSprint: database of computational hot spots in protein interfaces. Nucleic Acids Res. 36, D662-D666 (2008).

36 Keskin O, Ma B, Nussinov R. Hot regions in protein-protein interactions: the organization and contribution of structurally conserved hot spot residues. J. Mol. Biol. 345, 1281-1294 (2005).

37 Keskin O, Gursoy A, Ma B, Nussinov R. Towards drugs targeting multiple proteins in a systems biology approach. Curr. Top. Med. Chem. 7, 943-951 (2007).

38 Keskin O, Ma B, Rogale K, Gunasekaran K, Nussinov R. Protein-protein interactions: organization, cooperativity and mapping in a bottom-up systems biology approach. Phys. Biol. 2, S24-S35 (2005).

39 Haliloglu T, Keskin O, Ma B, Nussinov R. How similar are protein folding and protein binding nuclei? Examination of 
vibrational motions of energy hot spots and conserved residues. Biophys. J. 88, 1552-1559 (2005).

40 Lipinski CA. Drug-like properties and the causes of poor solubility and poor permeability. J. Pharmacol. Toxicol. Methods 44, 235-249 (2000).

41 Swinney DC. Biochemical mechanisms of drug action: what does it take for success? Nat. Rev. Drug Discov. 3, 801-808 (2004).

42 Schnur DM. Recent trends in library design: 'rational design' revisited. Curr. Opin. Drug Discov. Devel. 11, 375-380 (2008).

43 Shang S, Iwadare H, Macks DE, Ambrosini LM, Tan DS. A unified synthetic approach to polyketides having both skeletal and stereochemical diversity. Org. Lett. 9, 1895-1898 (2007).

44 Shang S, Tan DS. Advancing chemistry and biology through diversity-oriented synthesis of natural product-like libraries. Curr. Opin. Chem. Biol. 9, 248-258 (2005).

45 Kapetanovic IM. Computer-aided drug discovery and development (CADDD): in silico-chemico-biological approach. Chem. Biol. Interact. 171, 165-176 (2008).

46 Rester U. From virtuality to reality-virtual screening in lead discovery and lead optimization: a medicinal chemistry perspective. Curr. Opin. Drug Discov. Devel. 11, 559-568 (2008).
47 Morelli X, Rigby AC. Acceleration of the drug discovery process: a combinatorial approach using NMR spectroscopy and virtual screening. Curr. Computer-Aid Drug Des. 3, 33-49 (2007).

48 Jain AN. Virtual screening in lead discovery and optimization. Curr. Opin. Drug Discov. Devel. 7, 396-403 (2004).

49 Kitchen DB, Decornez H, Furr JR, Bajorath $\mathrm{J}$. Docking and scoring in virtual screening for drug discovery: methods and applications. Nat. Rev. Drug Discov. 3, 935-949 (2004).

50 Shoichet BK. Virtual screening of chemical libraries. Nature 432, 862-865 (2004).

51 Roche O, Schneider P, Zuegge J et al. Development of a virtual screening method for identification of "frequent hitters" in compound libraries. J. Med. Chem. 45, 137-142 (2002).

52 Warren GL, Andrews CW, Capelli AM et al. A critical assessment of docking programs and scoring functions. J. Med. Chem. 49, 5912-5931 (2006).

53 Lehar J, Stockwell BR, Giaever G, Nislow C. Combination chemical genetics. Nat Chem. Biol. 4, 674-681 (2008).

54 Stockwell BR. Chemical genetic screening approaches to neurobiology. Neuron 36 , 559-562 (2002).
55 Cordier C, Morton D, Murrison S, Nelson A, O'Leary-Steele C. Natural products as an inspiration in the diversity-oriented synthesis of bioactive compound libraries. Nat. Prod. Rep. 25, 719-737 (2008).

56 Peuchmaur M, Wong YS. Expanding the chemical space in practice: diversity-oriented synthesis. Comb. Chem. High Throughput Screen. 11, 587-601 (2008).

57 Blackwell HE, Perez L, Stavenger RA et al. A one-bead, one-stock solution approach to chemical genetics: part 1. Chem. Biol. 8, 1167-1182 (2001).

58 Clemons PA, Koehler AN, Wagner BK et al. A one-bead, one-stock solution approach to chemical genetics: part 2. Chem. Biol. 8, 1183-1195 (2001).

59 Pellecchia M. Solution nuclear magnetic resonance spectroscopy techniques for probing intermolecular interactions. Chem. Biol. 12, 961-971 (2005).

60 Pellecchia M, Becattini B, Crowell KJ et al. NMR-based techniques in the hit identification and optimisation processes. Expert Opin. Ther. Targets. 8, 597-611 (2004).

61 Pellecchia M, Sem DS, Wuthrich K. NMR in drug discovery. Nat. Rev. Drug Discov. 1, 211-219 (2002).

62 Zartler ER, Shapiro MJ. Fragonomics: fragment-based drug discovery. Curr. Opin. Chem. Biol. 9, 366-370 (2005). 